each biologic-treated patient with RA $(n=53,214)$ by sex, age, and geographical region. A patient could participate to several of these exposure cohorts. For each patient, follow-up under a cohort ended either with an outcome event (GI perforation identified in the NPR as an ICD10 code from a predefined list) or with the first of any of the following censoring events: emigration from Sweden, death, transition to another cohort, discontinuation of treatment $(+90 /+180$ days lag time) or end of study period. Crude incidence rates were tabulated for each cohort and adjusted hazard ratios (HR) and 95\% confidence intervals were estimated in multivariable Cox regressions, controlling for baseline differences. The final adjusted model included the following covariates: sex, age, line of biologic treatment, disease characteristics, co-medication at treatment start, co-morbidities and a history of GI perforation.

Results: We found 31 Gl perforations among 18604 person-years (pyr) exposed to TNFi, and $31 \mathrm{Gl}$ perforations among 10947 pyr exposed to non-TNFi, corresponding to crude incidence rates of 1.67 and 2.83 per 1000 pyr, respectively. The crude incidence rate among the biologics-naïve was 2.54 while among the general population comparators it was 0.94 . The rate of $\mathrm{Gl}$ perforations remained higher in patients with RA compared to the general population after adjustment for patient characteristics, HR of $1.78(95 \% \mathrm{Cl}: 1.44$ to 2.17$)$, whereas the seemingly increased rate among bionaïve and non-TNFi users vs TNFi was largely explained by differences in age and disease history at start of follow-up, with adjusted HRs of $1.10(0.68-1.78)$ for TNFi vs bionaïve and $1.10(0.63-1.91)$ for TNFi vs non-TNFi, respectively.

Conclusions: Although patients with RA had a higher rate of $\mathrm{GI}$ perforations than matched general population comparators, no significant differences in risk remained between bionaïve, TNFi or non-TNFi treated RA patients after adjusting for baseline patient characteristics.

Disclosure of Interest: None declared

DOI: 10.1136/annrheumdis-2018-eular.6831

\begin{tabular}{|l|l}
\hline OP0232 & FEMALE GENDER AND POSITIVE RHEUMATOID \\
& FACTOR PREDICT LOW SERUM INFLIXIMAB LEVELS \\
& AND POSITIVE ANTI-DRUG ANTIBODIES, WHICH \\
& ASSOCIATE WITH TREATMENT FAILURE ON \\
INFLIXIMAB IN PATIENTS WITH EARLY RHEUMATOID \\
ARTHRITIS. REPORT FROM THE SWEFOT TRIAL \\
POPULATION
\end{tabular}

K. Hambardzumyan ${ }^{1}$, C. Hermanrud ${ }^{1}$, P. Marits ${ }^{1}$, N. Vivar ${ }^{1}$, S. Ernestam ${ }^{1}$, J. K. Wallman ${ }^{2}$, R.F. van Vollenhoven ${ }^{1,3}$, A. Fogdell-Hahn ${ }^{1}$, S. Saevarsdottir ${ }^{1}$, on behalf of SWEFOT trial group. ${ }^{1}$ Karolinska Institutet, Stockholm; ${ }^{2}$ Lund University, Lund, Sweden; ${ }^{3}$ Amsterdam Rheumatology and Immunology Center, Amsterdam, Netherlands

Background: Tumour necrosis factor (TNF) inhibitors, with infliximab (IFX) first on the market, have revolutionised treatment of patients with rheumatoid arthritis (RA). However, in a substantial proportion of patients, they lose efficiency, and up to $44 \%$ of patients have been found to develop anti-drug antibodies (ADA), leading to low serum IFX (sIFX) levels. Despite this, sIFX measurement is still rarely used for clinical decision making, and standardised clinical threshold titre levels have not been clearly defined.

Objectives: In an early RA trial adding IFX to methotrexate (MTX) in patients not achieving low disease activity (LDA=DAS28 $\leq 3.2$ ) after 3 months monotherapy, we studied whether SIFX or ADA were associated with treatment outcome, and whether easily available baseline parameters predicted ADA development.

Methods: Of IFX-treated SWEFOT patients $(n=128), 101$ had available serum samples at follow-up, which were analysed for sIFX levels at 3, 9 and 21 months (routine ELISA). Samples with undetectable sIFX $(<0.2 \mu \mathrm{g} / \mathrm{ml})$ were analysed further for ADA using direct ELISA with plate-bound TNF. Primary and secondary outcome measures were LDA and remission (DAS28 <2.6) at 21 months. Clinical and demographic characteristics of patients at start of IFX therapy (baseline) were tested as potential predictors of ADA development, using uni- and multivariate logistic regression.

Results: At 3, 9 and 21 months from IFX add-on to MTX, 15\%, 23\% and $28 \%$ of patients, respectively, had undetectable sIFX, and 34\% were ever ADA-positive. Significantly higher proportion of patients achieved LDA among those with detectable sIFX, versus undetectable sIFX and positive ADA ( $67 \%$ vs $26 \%, p=0.002$, figure $1 \mathrm{~A})$, with similar difference for remission $(47 \%$ vs $11 \%, p=0.004$, figure $1 B)$. When sIFX levels were further stratified into $<0.2,0.2-5.0,5.0-10.0$ and $>10 \mu \mathrm{g} /$ $\mathrm{ml}$, there was a significant trend across the groups in achievement of LDA $(30 \%$, $65 \%, 70 \%$ and $83 \%$ respectively, $p=0.008$, figure $1 \mathrm{C})$ or remission $(10 \% ; 41 \%$, $52 \%$ and $67 \%$, respectively, $p=0.004$, figure 1D). Women had undetectable sIFX at 21 months more often than men ( $35 \%$ vs $7 \%, p=0.006)$. In multivariate logistic regression analysis, the following baseline characteristics were significant predictors of ever ADA-positivity: female gender, RF-positivity, higher tender joint count, erythrocyte sedimentation rate and lower health assessment questionnaire score (data not shown).
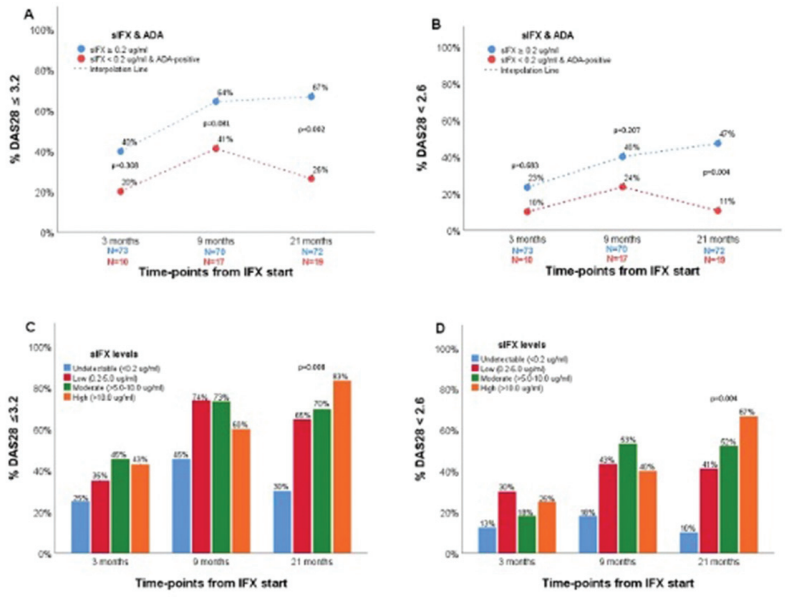

Abstract OP0232 - Figure 1 Clinical outcome of patients at 3, 9 and 21 months stratified for SIFX and ADA status at the same time points. Proportion of patients in LDA (A) and remission (B) among patient with detectable sIFX level (blue dots) and ADA positive patients with undetectable sIFX levels (red dots). Proportion of patients in LDA (C) and remission (D) among four strata of patients according to SIFX levels undetectable $(<0.2 \mu \mathrm{g} /$ $\mathrm{ml})$ - blue bars, low $(0.2-5.0 \mu \mathrm{g} / \mathrm{ml})$ - red bars, moderate $(>5.0-10.0 \mu \mathrm{g} / \mathrm{ml})$ - green bars, and high $(>10.0 \mu \mathrm{g} / \mathrm{ml})-$ orange bars.

Conclusions: In early RA patients receiving add-on IFX therapy, ADA-positivity or lower serum IFX levels were associated with a higher risk of not reaching treatment targets, that is LDA or remission. RF positivity and female gender, factors known to be associated with worse clinical outcomes, predicted development of ADA.

Disclosure of Interest: K. Hambardzumyan: None declared, C. Hermanrud: None declared, P. Marits: None declared, N. Vivar: None declared, S. Ernestam: None declared, J. Wallman Consultant for: AbbVie, Celgene, Eli Lilly, Novartis, UCB, R. van Vollenhoven Grant/research support from: AbbVie, BMS, GSK, Pfizer, UCB, Consultant for: AbbVie, AstraZeneca, Biotest, BMS, Celgene, GSK Janssen, Lilly, Novartis, Pfizer, UCB, A. Fogdell-Hahn Grant/research support from: Pfizer, S. Saevarsdottir: None declared DOI: 10.1136/annrheumdis-2018-eular.2877

\section{OP0233 LONG-TERM SAFETY OF ADALIMUMAB IN ADULT PATIENTS FROM GLOBAL CLINICAL TRIALS ACROSS MULTIPLE INDICATIONS: AN UPDATED ANALYSIS IN 29,987 PATIENTS REPRESENTING 56,951 PATIENT- YEARS}

G. Burmester ${ }^{1}$, R. Panaccione ${ }^{2}$, K. Gordon ${ }^{3}$, J. Rosenbaum 4 , D. Arikan ${ }^{5}$, W. Lau ${ }^{5}$, R. Tarzynski-Potempa ${ }^{5}{ }^{1}$ Charité - University Medicine Berlin, Berlin, Germany; ${ }^{2}$ University of Calgary, Calgary, Canada; ${ }^{3}$ Medical College of Wisconsin, Milwaukee; ${ }^{4}$ Oregon Health and Science University and Legacy Devers Eye Institute, Portland; ${ }^{5}$ AbbVie Inc., North Chicago, USA

Background: Adalimumab is an anti-tumour necrosis factor- $\alpha$ (TNF- $\alpha$ ) agent indicated for the treatment of immune-mediated diseases. The long-term safety of adalimumab was previously reported in 23458 patients representing up to 12 years of clinical trial exposure in rheumatoid arthritis (RA), juvenile idiopathic arthritis, ankylosing spondylitis (AS), psoriatic arthritis (PSA), plaque psoriasis $(\mathrm{Ps})$, and Crohn's disease (CD).

Objectives: Here we report an updated analysis examining the long-term safety of adalimumab in adult patients with RA, AS, non-radiographic axial spondyloarthritis (nr-axSpA), peripheral SpA (pSpA), PsA, Ps, hidradenitis suppurativa (HS), $\mathrm{CD}$, ulcerative colitis (UC), and non-infectious uveitis (UV).

Methods: Here we report an updated analysis examining the long-term safety of adalimumab in adult patients with RA, AS, non-radiographic axial spondyloarthritis (nr-axSpA), peripheral $\mathrm{SpA}(\mathrm{pSpA}), \mathrm{PsA}, \mathrm{Ps}$, hidradenitis suppurativa (HS), $\mathrm{CD}$, ulcerative colitis (UC), and non-infectious uveitis (UV). 